\section{Complete endoscopic closure of a large gastric defect with endoloop and endoclips after complex endoscopic submucosal dissection}

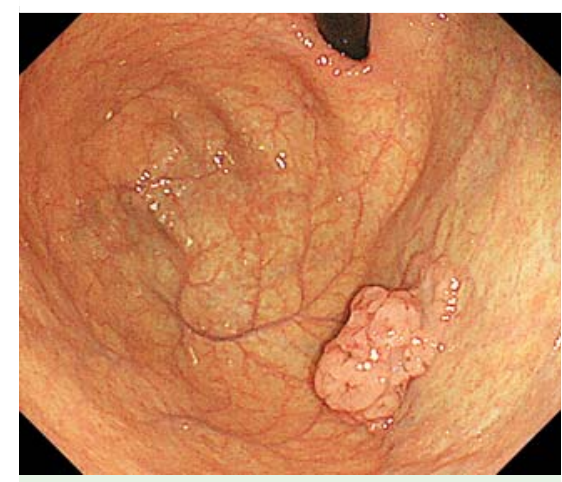

Fig. 1 A protruded lesion found on the anterior side of the greater curvature of the gastric fundus in an 83-year-old woman undergoing esophagogastroduodenoscopy for anemia.

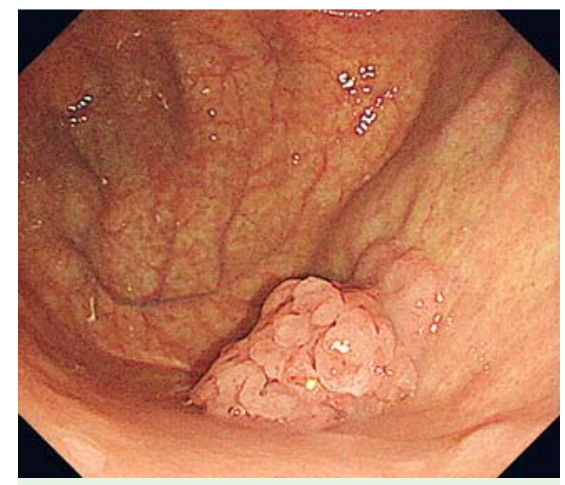

Fig. 2 The lesion was easily submerged under water in deflation view.

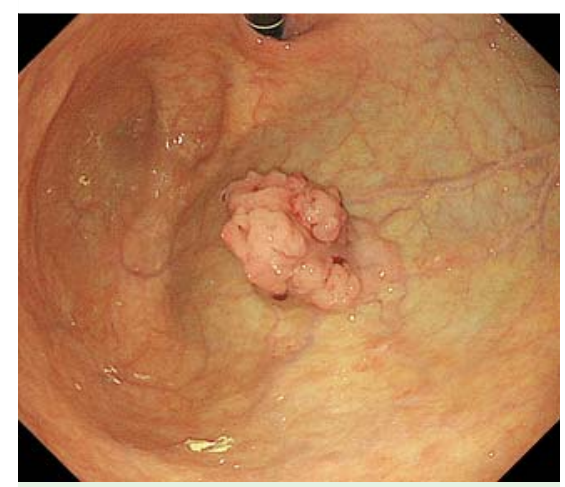

Fig. 3 The lesion was shifted away from pooled water with the patient in the supine position.

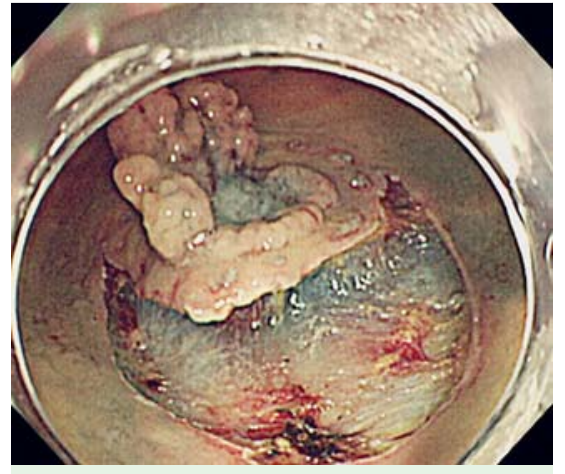

Fig.4 Partial mucosal incision and submucosal dissection were first done from the distal side in retroflexed view.

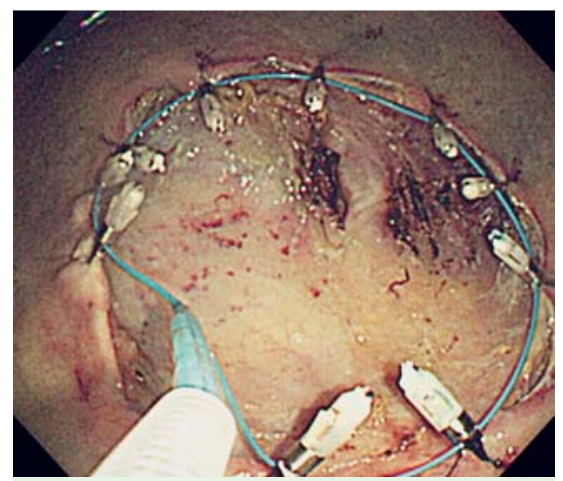

Fig. 5 A double-channel gastroscope was used to place an endoloop along the edge of the mucosal defect and fix it with hooked endoclips. The mucosal defect was then successfully closed by tightening the fixed endoloop.

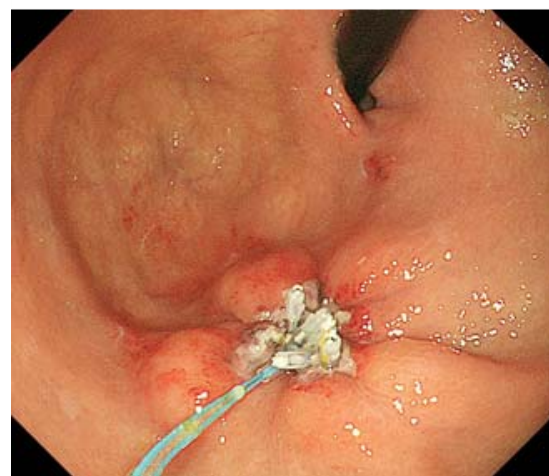

Fig. 6 Second-look endoscopy on day 2 after endoscopic submucosal dissection showed sustained complete closure with the tightened endoloop.
An 83-year-old woman on long-term warfarin and aspirin therapy after aortic valve replacement and coronary stent intervention underwent esophagogastroduodenoscopy for anemia. A protruded lesion was found on the anterior side of the greater curvature of the gastric fundus $(\bullet$ Fig.1). This lesion was easily submerged under water in deflation view ( $\bullet$ Fig. 2). The patient opted for endoscopic submucosal dissection (ESD).

A double-channel gastroscope with a double-bending function provided by independent angulation of two separate sections (GIF-2TQ260M; Olympus, Tokyo, Japan) was used to allow a close approach to the lesion. Because the potential point of bleeding would be obscured in the event of massive bleeding, the patient's position was changed from left lateral to supine, and the procedure was conducted while the patient was under general anesthesia. With the change of position, the lesion was shifted away from the greater curvature ( Fig.3).

Partial mucosal incision and submucosal dissection were first done from the distal side in retroflexed view ( Fig.4). This allowed good exposure of the distal submucosa and visualization of the point of bleeding with traction toward the proximal side [1]. Therefore, hemostasis subsequently became easier, and ESD was completed without severe bleeding.

After prophylactic coagulation of the mucosal defect, an endoloop was opened through the first channel, and an endoclip was prepared through the other channel. We hooked the endoclip onto the endoloop and then used the hooked endoclip to anchor the endoloop. Additional endoclips were used to fix the endoloop along the edge of the mucosal defect ( $\bullet$ Fig.5). The mucosal defect was successfully closed by tightening the fixed endoloop ( $\bullet$ Video 1), as in the report of Matsuda et al. [2].

Second-look endoscopy on day 2 after ESD showed sustained complete closure with the tightened endoloop ( Fig.6). The patient was discharged 5 days after ESD without any complication. Examination of the resected specimen indicated a well-differentiated tubular adenocarcino$\mathrm{ma}, 29 \mathrm{~mm}$ in size, with a depth to the deep submucosa and a free margin.

This case highlights the following technical points: First, it is quite important to maintain an adequate operative field to conduct a difficult gastric ESD. Second, appropriate accessories and scopes should be available to close large defects. Delayed 


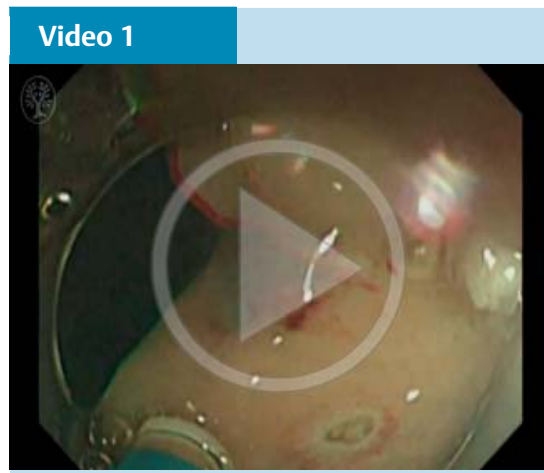

Difficult gastric endoscopic submucosal dissection (ESD) followed by complete closure with an endoloop and endoclips. Partial mucosal incision and submucosal dissection were first done from the distal side in retroflexion with a Dual Knife (KD-650Q; Olympus, Tokyo, Japan) and IT knife 2 (KD-611L; Olympus). This procedure allowed good exposure of the distal submucosa and visualization of the point of bleeding with traction toward the proximal side. Subsequently, hemostasis became easier, and ESD was completed without severe bleeding. After prophylactic coagulation of the mucosal defect, an endoloop was opened through one channel, and an endoclip was prepared through the other channel. We hooked the endoclip onto the endoloop and used it to anchor the endoloop. Several additional endoclips were then used to fix the endoloop along the edge of the mucosal defect. The mucosal defect was successfully closed by tightening the fixed endoloop. bleeding after gastric ESD is more common in patients on anticoagulant therapy $[3,4]$, and complete closure of the mucosal defect with an endoloop and endoclips can prevent delayed bleeding, as shown in this case.

Endoscopy_UCTN_Code_TTT_1AO_2AC

Competing interests: None

Seiichiro Abe, Ichiro Oda, Genki Mori, Satoru Nonaka, Haruhisa Suzuki, Shigetaka Yoshianaga, Yutaka Saito

Endoscopy Division, National Cancer Center Hospital, Tokyo, Japan

\section{Acknowledgement $\nabla$}

The authors are grateful to Hiroyuki Takamaru, MD, PhD, and Masau Sekiguchi, MD, of the Endoscopy Division, National Cancer Center Hospital, Tokyo, Japan, for their kind support during the preparation of this report. In addition, they would like to say thank Klaus Mönkemüller MD, PhD, of Basil I. Hirschowitz Endoscopic Center of Excellence, Division of Gastroenterology and Hepatology, University of Alabama at Birmingham, Alabama, USA, for editing this paper.

\section{References}

1 Mori G, Nonaka S, Oda I et al. Novel strategy of endoscopic submucosal dissection using an insulation-tipped knife for early gastric cancer: near-side approach method. Endosc Int Open (in press)

2 Matsuda T, Fujii T, Emura F et al. Complete closure of a large defect after EMR of a lateral spreading colorectal tumor when using a two-channel colonoscope. Gastrointest Endosc 2004; 60: 836-838

3 Koh R, Hirasawa K, Yahara $S$ et al. Antithrombotic drugs are risk factors for delayed postoperative bleeding after endoscopic submucosal dissection for gastric neoplasms. Gastrointest Endosc 2013; 78: 476-478

4 Takeuchi T, Ota $K$, Harada $S$ et al. The postoperative bleeding rate and its risk factors in patients on antithrombotic therapy who undergo gastric endoscopic submucosal dissection. BMC Gastroenterol 2013; 13: 136

Bibliography

Dol http://dx.doi.org/

10.1055/s-0034-1392594

Endoscopy 2015; 47: E374-E375

(c) Georg Thieme Verlag KG

Stuttgart · New York

ISSN 0013-726X

\section{Corresponding author}

\section{Seiichiro Abe, MD}

Endoscopy Division

National Cancer Center Hospital

5-1-1 Tsukiji

Chuo-ku

Tokyo 104-0045

Japan

Fax: +81-3-3542-3815

seabe@ncc.go.jp 